\title{
Coronary and cerebral air embolism: a rare complication of computed tomography-guided transthoracic lung biopsy
}

\author{
D. R. Smit • S. A. Kleijn • \\ W. G. de Voogt \\ Published online: 10 April 2013 \\ (C) The Author(s) 2013. This article is published with open access at Springerlink.com
}

\section{Case report}

A 71-year-old man with a history of excessive smoking was evaluated for an abnormal chest X-ray. He had no angina and no risk factors for coronary artery disease. Considering a high probability of lung cancer in this patient, computed tomography-guided transthoracic lung biopsy was performed by an experienced radiologist. A co-axial 18-gauge needle system was used to enter the lung and two core biopsy samples were obtained. During the procedure the patient was asked to inhale in order to reach the lesion. After the biopsy he developed mild haemoptysis followed by rapid loss of consciousness. After a couple of seconds the patient regained consciousness, was asymptomatic and did not show any signs of respiratory or haemodynamic complications. However, the ECG showed ST-segment elevation in leads I, II, aVL, aVF and V3-6 without reciprocal ST-segment depression suggestive of an acute myocardial infarction involving the distributions of more than one coronary artery (Fig. 1c). Review of the multi-slice CT images showed a mild pneumothorax, pulmonary haemorrhage, and air bubbles in both the circumflex coronary artery and descending aorta, diagnostic of an air embolism of the arterial system (Fig. 1a, b). He was

\section{R. Smit ( $\square)$}

Department of Cardiology, VU University Medical Center, De Boelelaan 1117,

$1081 \mathrm{HV}$, Amsterdam, the Netherlands

e-mail: d.smit@vumc.nl

\section{S. A. Kleijn}

Department of Internal Medicine, Sint Lucas Andreas Hospital, Amsterdam, the Netherlands

W. G. de Voogt

Department of Cardiology, Sint Lucas Andreas Hospital,

Amsterdam, the Netherlands placed in a mild Trendelenburg position and was administered $100 \%$ high-flow non-rebreathing oxygen. Anticoagulation therapy was not initiated due to the pulmonary haemorrhage. Electrocardiography showed complete resolution of the ST-segment elevation after $20 \mathrm{~min}$. However, $3 \mathrm{~h}$ later he developed seizures likely due to air embolisation to the cerebral circulation. A subsequent blood test showed cardiac troponin I elevation of $11.0 \mu \mathrm{g} / \mathrm{l}$, whereas echocardiography demonstrated hypokinesis in the distal inferior and anteroseptal regions. The patient was eventually discharged after 1 week without any cardiac, pulmonary or neurological symptoms.

\section{Discussion}

Computed tomography-guided transthoracic lung biopsy is a common clinical procedure for the diagnosis of a broad range of pulmonary pathological conditions. Mild self-limiting pneumothorax and pulmonary haemorrhage are common complications of this procedure. With incidences between $0.01 \%$ and $0.21 \%$, the occurrence of an air embolism in the left atrium, left ventricle, or systemic circulation during a percutaneous core needle biopsy of the lung is a rare, but potentially fatal complication [1]. The incidence might be underestimated by missing systemic air embolisms in patients without cardiac or cerebral symptoms [2]. Freund et al. recently illustrated that although rare, radiological incidence of a systemic air embolism during a percutaneous core needle biopsy of the lung was $3.8 \%$ (23/610 patients), whereas the clinically apparent incidence was $0.49 \%$ [1]. Our patient had a very unusual presentation of myocardial infarction and documented air embolisation in the coronary circulation and aorta with loss of consciousness possibly due to a cerebral air embolus. He 
Fig. 1 a CT image shows a CTguided transthoracic lung biopsy with the biopsy needle (white arrow) entering the left lung. b CT image showing a mild pneumothorax (white arrow), pulmonary haemorrhage and air bubbles in both the circumflex coronary artery and descending aorta (black arrows). c ECG showing marked ST-segment elevation in leads I, II, aVL, aVF and V3-6

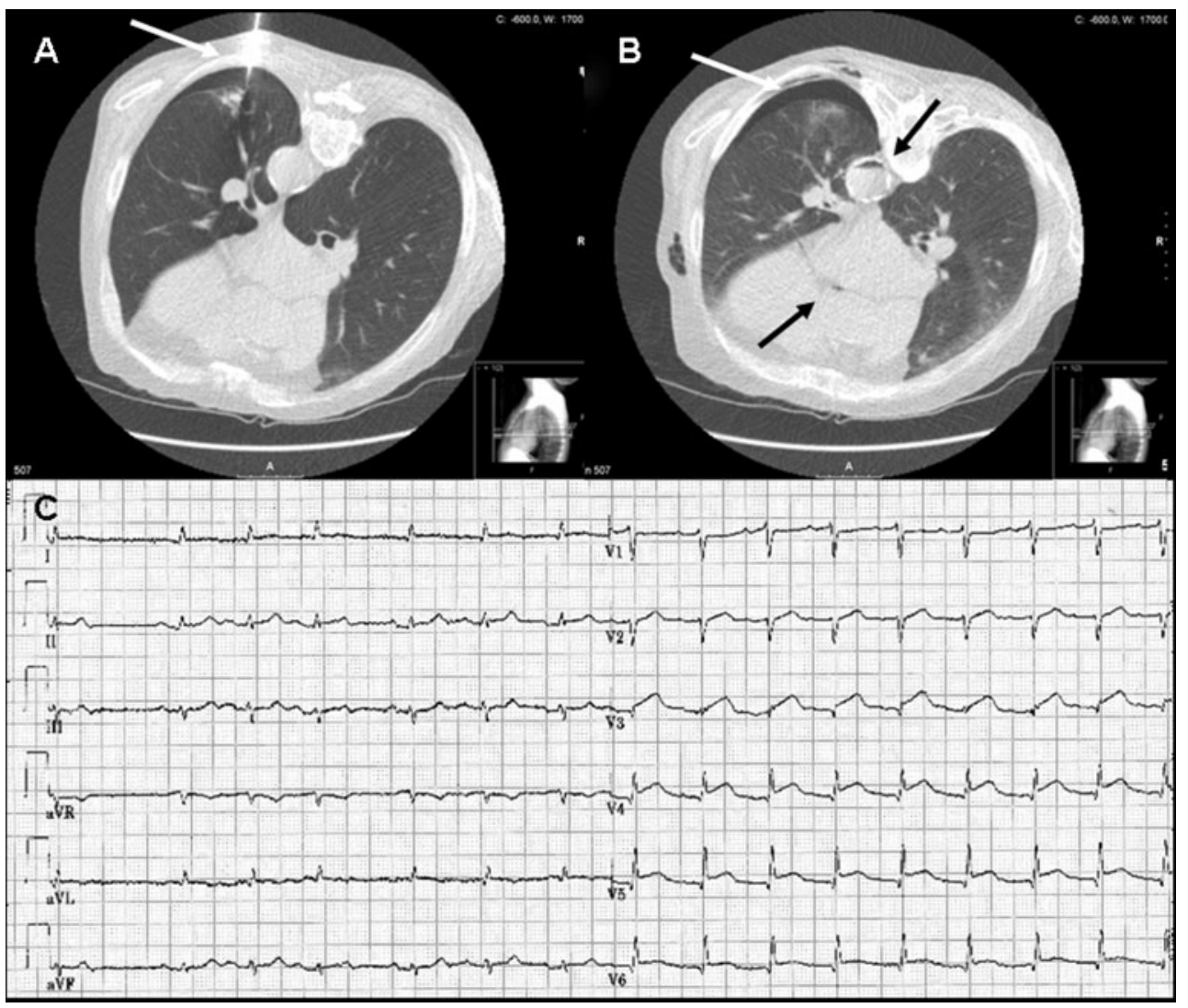

later experienced seizures due to an air embolus to the cerebral circulation, although this could not be proven [3].

The mechanism for air embolisation has been explained by two different mechanisms. First, when the tip of the biopsy needle is lodged in a pulmonary vein, air embolisation can occur with the removal of the inner stylet during rapid inspiration due to the atmospheric pressure exceeding the pulmonary venous pressure. Second, when a needle simultaneously transverses an air-containing space and adjacent pulmonary vein, a fistula can occur and air will enter the vein when the alveolar air pressure is greater than the pulmonary venous pressure, for example during coughing [4-6]. We did not treat the patient with hyperbaric oxygen because the symptoms were transient. Trendelenburg position and $100 \%$ oxygen has had a favourable outcome in other cases $[4,5,7,11]$. Air embolisation has been described to reduce perfusion distal to the obstruction as well as induce an inflammatory response to the bubble [7]. There is evidence that heparin may be beneficial in the treatment of gas embolism [8]. We concluded that this was a self-limiting complication and did not administer heparin or other antiplatelet therapy due to the pulmonary haemorrhage [9-12].

\section{Conclusion}

Our case illustrates the possible fatal complication that due to its low incidence is relatively underdiagnosed. Broader awareness of this life-threatening complication of CT-guided lung biopsy should allow fast initial recognition and management by interventional radiologists and cardiologists for improved outcome.

Funding None.

Conflict of interests None declared.

Open Access This article is distributed under the terms of the Creative Commons Attribution License which permits any use, distribution, and reproduction in any medium, provided the original author(s) and the source are credited.

\section{References}

1. Freund MC, Petersen J, Goder KC, et al. Systemic air embolism during percutaneous core needle biopsy of the lung: frequency and risk factors. BMC Pulm Med. 2012;12:2.

2. Hiraki T, Fujiwara H, Sakurai J, et al. Nonfatal systemic air embolism complicating percutaneous CT-guided transthoracic needle biopsy: four cases from a single institution. Chest. 2007;132:684-90.

3. Six AJ, Backus BE, Kingma A, et al. Consumption of diagnostic procedures and other cardiology care in chest pain patients after presentation at the emergency department. Neth Heart J. 2012;20:499-504. 
4. Wu CC, Maher MM, Shepard JA, et al. Complications of CT-guided percutaneous needle biopsy of the chest: prevention and management. AJR Am J Roentgenol. 2011;196:67882.

5. Hsi DH, Thompson TN, Fruchter A, et al. Simultaneous coronary and cerebral air embolism after CT-guided core needle biopsy of the lung. Tex Heart Inst J. 2008;35:472-4.

6. Wu YF, Huang TW, Kao CC, et al. Air embolism complicating computed tomography-guided core needle biopsy of the lung. Interact Cardiovasc Thorac Surg. 2012;14:771-2.

7. Moon RE. Gas embolism. In: Oriani G, Marroni A, Wattel F, editors. Handbook on hyperbaric medicine. Milan: Springer; 1996. p. 229-48.
8. Ryu KH, Hindman BJ, Reasoner DK, et al. Heparin reduces neurological impairment after cerebral arterial air embolism in the rabbit. Stroke. 1996;27:303-10.

9. Verheugt FW. Antithrombotic therapy in heart failure. Neth Heart J. 2012;20:176-8.

10. van der Zee PM, Verberne HJ, Cornel JH, et al. GRACE and TIMI risk scores but not stress imaging predict long-term cardiovascular follow-up in patients with chest pain after a rule-out protocol. Neth Heart J. 2011;19:324-30.

11. Ulucay A, Aksoy MF, Sahin E. Transthoracic echocardiography may show saddle pulmonary embolism. Neth Heart J. 2011;19:531.

12. Hoendermis ES. Pulmonary arterial hypertension: an update. Neth Heart J. 2011;19:514-22. 